\title{
Asimetría bilateral de la forma de las valvas y posición espacial en matrices del chorito Perumytilus purpuratus (Lamarck, 1819) (Bivalvia: Mytilidae)
}

\author{
Bilateral asymmetry of shell shape and spatial position in matrices of the mussel \\ Perumytilus purpuratus (Lamarck, 1819) (Bivalvia: Mytilidae)
}

\author{
CAROLINA BRIONES ${ }^{1,2} \&$ RICARDO GUIÑEZ ${ }^{1,3, *}$
}

\author{
${ }^{1}$ Instituto de Investigaciones Oceanológicas, Facultad de Recursos del Mar, Universidad de Antofagasta, Casilla 170, \\ Antofagasta, Chile \\ ${ }^{2}$ Dirección actual: Facultad de Ciencias del Mar, Universidad de Valparaíso, Chile \\ ${ }^{3}$ Center for Advanced Studies in Ecology \& Biodiversity. Facultad de Ciencias Biológicas, Pontificia Universidad Católica \\ de Chile, Casilla 114-D, Santiago, Chile \\ *Autor para correspondencia; e-mail: rguinez@bio.puc.cl
}

\begin{abstract}
RESUMEN
De las distintas formas de la asimetría bilateral, la asimetría direccional (AD) ha recibido considerablemente menos atención en la literatura que la asimetría fluctuante (AF). Si bien existen buenos ejemplos de AD conspicua en algunos grupos de moluscos (e.g., Ostreidae y Pectinidae), para otros como los mitílidos la AD no ha sido reportada. En matrices del chorito Perumytilus purpuratus de tres localidades del intermareal rocoso de Chile norte y central, comparando individuos de los estratos superior e inferior, y usando dos índices de forma de la valva, reportamos evidencia de AD en relación con la forma de las valvas, tendiendo la derecha a ser consistentemente más elongada que la valva izquierda. Sin embargo, no solo en estratos inferiores de la matriz los choritos tienden a tener valvas menos elongadas sino que también tienden a ser más asimétricas, en relación con el estrato superior. Los resultados sugieren que el cambio de forma y de asimetría podría estar siendo modulado por la densidad o la estratificación. Las explicaciones causales de este fenómeno están abiertas a la investigación.
\end{abstract}

Palabras clave: asimetría direccional, asimetría fluctuante, chorito, mitílido, forma de la concha.

\begin{abstract}
In the literature, less attention has been paid to the study of directional asymmetry (DA) than fluctuating asymmetry (FA). Although there are good examples of DA in mollusks (e.g., Ostreidae and Pectinidae), for some taxa, such as mussels, DA has not been reported. We examined asymmetry in two shell shape traits of the mussel Perumytilus purpuratus, in the rocky intertidal zone of three localities in central and northern Chile. We compared shell asymmetry between mussels occupying different positions in the matrices or mussel beds (i.e., upper versus lower stratum). We observed shell shape DA for mussels occupying both strata, with the right valve being consistently less elongated than the left valve. However, for individuals in the lower strata the mussel valves tended to be more elongated, and also more asymmetrical, relative to mussels from the upper layer of the matrix. These results suggest that changes in the shape and asymmetry of the mussel valves may be modified by density and stratification in the beds. The causal explanations of this phenomenon are open to further research.
\end{abstract}

Key words: directional asymmetry, fluctuating asymmetry, mussel, mytilid, shell shape.

\section{INTRODUCCIÓN}

La asimetría de rasgos bilaterales ha sido ampliamente reconocida como una estimación de la incapacidad de los organismos para mantener su desarrollo normal, contra ruidos o perturba- ciones de origen ambiental y como un reflejo de la inestabilidad del desarrollo (Palmer \& Strobeck 1992, Lens \& Van Dongen 2000). Sin embargo, no existe consenso en torno a cuál de los principales tipos de asimetría bilateral (AB) (i.e., fluctuante, direccional o antisimetría) re- 
flejaría la inestabilidad del desarrollo. De estos tres tipos de $\mathrm{AB}$ es la asimetría fluctuante (AF) la que ha recibido la mayor atención (Pither \& Taylor 2000, Palmer \& Strobeck 2003). La AF se define como las desviaciones sutiles y aleatorias de la simetría de rasgos bilateralmente simétricos (Palmer \& Strobeck 2003) y ha sido correlacionada con estrés fisiológico causado por condiciones ambientales extremas (e.g., Beardmore 1960, Gest et al. 1986, Hosken et al. 2000). Esto ha estimulado su uso potencial como una herramienta para el seguimiento de los niveles de estrés en poblaciones naturales y como un indicador de la calidad individual o la adecuación darwiniana (e.g., Graham et al. 1993, Palmer 1996a, Møller 1997, Leung et al. 2000, Ditchkoff et al. 2001, Fréchette \& Daigle 2002, Fréchette et al. 2003). La antisimetría corresponde al caso en que los rasgos presentan una distribución bimodal con promedio cero (Palmer \& Strobeck 1986), y por otra parte, la asimetría direccional (AD) ocurre cuando el valor de un lado del rasgo es consistentemente más grande que el otro (distribución normal con promedio distinto de cero), incluyendo situaciones en que estas diferencias son sutiles. La $\mathrm{AD}$ ha recibido considerablemente menor atención que la AF (Leary \& Allendorf 1989, Kraak 1997, Lens \& Van Dongen 2000, Pither \& Taylor 2000). El grado del sesgo de la literatura en reportar la AD no se conoce, pero posiblemente es significativo (Pither \& Taylor 2000). Este sesgo puede haber sido incrementado por el hecho de que se considera a la $\mathrm{AD}$ con una base genética significativa (Palmer \& Strobeck 1986, Palmer 1994) y por lo tanto, no apta como una medida de la inestabilidad del desarrollo (Lens \& Van Dongen 2000). Sin embargo, evidencia creciente sugiere que los tres tipos de asimetría debieran ser considerados como procesos dinámicamente interrelacionados (Lens \& Van Dongen 2000). En moluscos bivalvos existen grupos con AD conspicua, por ejemplo, los individuos pueden presentar la valva con que se adhieren al sustrato más pequeño (e.g., Anomiacea y Pectinacea) o de mayor tamaño (e.g., Ostreacea) (ver revisión de Palmer 1996b). En otros como el pectínido Chlamys islandica y la ostra Crassostrea gigas, se han documentado casos de AF asociados a diferentes tasas de mortalidad (Fréchette \& Daigle 2002, Fréchette et al. 2003). Sin embargo, en mitílidos no existen reportes conocidos por nosotros sobre la existencia de alguna forma de asimetría bilateral.

Una de las características notables del chorito intermareal Perumytilus purpuratus (Lamarck, 1819) y de muchas otras especies de mitílidos, es su tendencia a formar agregaciones de individuos altamente densas, conformando usualmente matrices multiestratificadas (Suchanek 1986, Alvarado \& Castilla 1996, Guiñez \& Castilla 1999), en cuyo interior ocurre una intensa competencia intraespecífica mediada por restricciones de espacio o energéticas (Fréchette \& Lefaivre 1990, Guiñez 1996, Guiñez \& Castilla 1999). Esto expone a los individuos a un ambiente altamente estresante en el que la sobrevivencia y el crecimiento disminuyen con el incremento de la densidad y la estratificación (Bertness \& Grosholz 1985, Guiñez \& Castilla 1999). En algunos taxa marinos se han comunicado cambios de forma asociados al incremento de la densidad, tales como ostras (Chinzei et al. 1982), almejas (Cigarría \& Fernández 1998), cirripedios (Bertness et al. 1998) y tunicados (Paine \& Suchanek 1983, Castilla et al. 2000). En choritos, también se han reportado cambios de forma asociados a la densidad, por ejemplo en Mytilus edulis los individuos se hacen más elongados (Seed 1968, Alunno-Bruscia et al. 2001), y en $P$. purpuratus hemos observado la tendencia contraria, tendiendo en altas agregaciones (multiestratificadas) a ser menos elongados (R. Guiñez, resultados no publicados). Sin embargo, los efectos de la competencia intraespecífica también pueden depender de la posición de los individuos en la matriz. En efecto, en el chorito $M$. edulis se ha demostrado experimentalmente que los individuos en los bordes de las agregaciones crecen y producen mayor cantidad de tejidos reproductivos (Okamura 1986) y en $P$. purpuratus, los individuos localizados en el estrato superior son menos afectados por la competencia intraespecífica que aquellos ubicados en estratos inferiores, presentando además diferencias en la distribución de tamaños y en la cantidad de reclutas (Alvarado \& Castilla 1996, Guiñez \& Castilla 1999). Es esperable, entonces, que los individuos ubicados en estratos inferiores de la matriz estén sometidos a condiciones más extremas y estresantes que aquellos ubicados en el estrato superior. En consecuencia, también sería esperable que los individuos no solo presenten cambios de forma en relación con el estrato en el que se 
ubican, sino que también puedan presentar diferente asimetría bilateral en relación con los rasgos de la forma en ambas valvas.

El propósito de este trabajo es comunicar los primeros resultados de una investigación en desarrollo sobre las evidencias de la existencia de asimetría bilateral de las valvas de $P$. purpuratus y su posible relación con la posición de los choritos dentro de la matriz.

\section{MATERIALES Y MÉTODOS}

\section{Recolección de las muestras}

Entre marzo y mayo de 2002 se tomaron azarosamente 36 muestras de choritos de la especie Perumytilus purpuratus, desde la franja intermareal media (Castilla 1981). Las muestras fueron obtenidas de matrices del chorito con $100 \%$ de cobertura ubicadas sobre rocas planas con pendientes menores a $10^{\circ}$, desde las localidades: (a) Punta de Tralca $\left(33^{\circ} 26^{\prime} \mathrm{S}, 71^{\circ} 43^{\prime} \mathrm{O}, \mathrm{n}=23\right.$ ) ubicado en la costa de Chile central, y (b) El Way $\left(23^{\circ} 45^{\prime} 02.5^{\prime \prime} \mathrm{S}, 70^{\circ} 26^{\prime} 43.3^{\prime \prime} \mathrm{O}, \mathrm{n}=5\right)$, y (c) Llacolén (3 km al norte de El Way, $\mathrm{n}=8$ ) ubicados al interior de la Bahía de Antofagasta (norte de Chile). Cada muestra se obtuvo removiendo, con ayuda de una espátula metálica, todos los choritos presentes en un área de $10 \times 10$ cm (Guiñez 1996, Guiñez \& Castilla 1999); previamente los choritos del estrato superior fueron pintados (Alvarado \& Castilla 1996, Guiñez 1996) para poder diferenciar posteriormente a los individuos de ambos estratos (superior versus inferior). Las muestras fueron almacenadas en bolsas plásticas (Ziploc), cuidadosamente etiquetadas, y congeladas a $-20{ }^{\circ} \mathrm{C}$ hasta su procesamiento y medición.

Para cada una de las muestras, se seleccionó al azar a 20 individuos de cada estrato (superior e inferior), intentando representar la distribución de tamaños de cada muestra entre los rangos de 7 a $37 \mathrm{~mm}$ de longitud máxima (los choritos de esta especie raramente superan los 41 mm; Alvarado \& Castilla 1996, Guiñez 1996, Guiñez \& Castilla 1999). Para la identificación de individuos de estratos inferiores se consideró solo a aquellos individuos con ausencia absoluta de pintura. Sin embargo, no en todos los casos se pudo colectar el máximo de 20 choritos de la capa inferior, aún más, de todas las muestras de Punta de Tralca solo se pudo obtener un total de 37 individuos de estratos infe- riores, debido a que el nivel de agregación en esta localidad es mucho menor que en Antofagasta (R. Guiñez, resultados no publicados). Posteriormente a su identificación, cada individuo fue cuidadosamente limpiado de todo rastro de pintura y de epibiontes.

\section{Mediciones}

Los choritos fueron medidos para ancho máximo $( \pm 0,005 \mathrm{~mm})$ y peso húmedo de tejidos blandos total y de cada valva $( \pm 0,0005 \mathrm{~g})$. Antes de procesar las valvas, se procedió a remover el ligamento de la charnela. Cada valva (izquierda y derecha) fue dispuesta sobre una cámara lúcida horizontal fija en posición estándar, con el umbo (extremo anterior) en posición sur y el extremo posterior en posición norte con la concavidad de la valva hacia abajo, fotografiada y digitalizada con una cámara fotográfica digital SONY TRV 530 (con una calidad de imagen de 1,30 megapixeles) instalada en un trípode a una distancia uniforme de $30 \mathrm{~cm}$. En cada fotografía se incluyó una moneda (de dimensiones conocidas) y una reglilla de $50 \mathrm{~mm}$ con el objeto de calibrar la medición posteriormente. Las imágenes fueron analizadas y procesadas usando el programa de libre acceso UTHSCSA ImageTool Versión 3.0 (desarrollado por University of Texas Health Science Center, San Antonio, Texas, USA, disponible desde Internet en la dirección: ddsdx.uthscsa.edu/dig/itdesc.html), usando medidas en milímetros y tres decimales de precisión. Para cada imagen se realizó la calibración espacial usando la reglilla de $50 \mathrm{~mm}$ de referencia, en posición perpendicular al objeto (esteoeste), y se ajustó manualmente el umbral de contraste hasta llevar el objeto a imagen en blanco y negro. Sobre este objeto (imagen de valva), junto con la moneda de dimensiones conocidas, se estimó la superficie, el diámetro, largo máximo y ancho máximo, y se calcularon dos índices de forma: índice de elongación (IELO) y de redondez (IRED). Para cada imagen se corrigieron las medidas obtenidas de acuerdo a las medidas lineales y de superficie estándar de la moneda. El índice de elongación (IELO) se estimó como la razón entre la longitud máxima y el ancho máximo, mientras mayor valor tiene IELO más alargado y menos ancho es el individuo. El índice de redondez (IRED) se calculó como $(4 \pi$ área) / Perímetro ${ }^{2}$, en este caso los valores del índice fluctúan entre cero y uno. Si el valor es 
uno entonces la forma del objeto (forma de la concha) es perfectamente circular, y si los valores se acercan a cero, la forma se aleja de una forma circular.

Tipos de asimetrías, error de medición y análisis estadístico

Para cuantificar el error de medición, se eligieron aleatoriamente 40 individuos de cada localidad y posición (estrato superior y estrato inferior), usando números al azar, los que se volvieron a medir (ver siguiente párrafo) de tal modo que en total se dispuso de seis muestras. En este trabajo solo se comunican los resultados de los análisis preliminares para estas seis muestras.

Estos individuos se midieron nuevamente a partir de la fotografía original, variando las siguientes fuentes posibles de error: amplificación de la imagen y recalibración de la medida espacial de la reglilla, también se varió manualmente el umbral de contraste antes de llevar la imagen a blanco y negro. Para cada valva se volvió a corregir las medidas lineales y de superficie, usando las medidas estándar de la moneda. De este modo para cada individuo y valva se tuvo dos medidas repetidas (M1 y M2). Para esta segunda medición solo se trabajó con el código de cada foto, con desconocimiento de si la valva era izquierda o derecha, y en distinto orden.

El error de medición se analizó mediante los métodos de Palmer \& Strobeck (1986), Palmer (1994) y Palmer \& Strobeck (2003), para lo que se aplicó un análisis de varianza de medidas repetidas mixto para cada muestra, usando el siguiente modelo estadístico: $Y_{i j k}=\mu+L_{i}+I_{j}$ $+L I_{i j}+k_{(i j)}$, donde $Y_{i j k}$ es la $k$ medida repetida del índice $Y$ (variable respuesta) dentro del lado $i$ del individuo $j, \mu$ es la media poblacional, $L_{i}$ es el efecto fijo del lado $i, I_{j}$ es el efecto aleatorio de individuo $j, L I_{i j}$ es el efecto aleatorio de la interacción del lado $i$ con el individuo $j, \mathrm{y}$ $e_{k(i j)}$ es el error residual (varianza de las medidas repetidas). Un efecto significativo del factor lado $(L)$ indica la presencia de asimetría direccional (AD) y permite poner a prueba la hipótesis de si la diferencia $\operatorname{Dif}_{-1}=$ lado derecho - lado izquierdo, es distinta de cero, esto es equivalente a realizar una prueba $t$ de Student de dos colas. Un efecto significativo del factor 'individuo' (I) indica que las diferencias entre individuos son mayores que el error de medición (EM), y un efecto significativo del término de interacción $L I$, es una prueba estadística para determinar si los distintos tipos de asimetrías son más grandes que el EM. Para determinar la probabilidad de rechazo de la hipótesis nula, se ajustó las probabilidades del error tipo I mediante la corrección secuencial de Bonferroni (Quinn \& Keough 2002).

Para verificar la ausencia de antisimetría (AS) se determinó que la distribución no fuera platicúrtica, mediante la puesta a prueba de que la curtosis $\left(g^{2}\right)$ de la distribución de Dif 1 fuera igual o mayor a cero. Antes de someter los datos a estos análisis se procedió a identificar datos extremos ('outliers') usando gráficos de dispersión de residuales de acuerdo a procedimientos estándar (Palmer \& Strobeck 2003). Los datos extremos no fueron incorporados en los análisis. Además de determinar si la asimetría promedio de una muestra particular fue distinta de cero, estuvimos interesados en ver si la dirección de la asimetría fue consistente entre las distintas muestras. Para ello usamos una prueba binomial, en que la hipótesis nula fue que los valores positivos y negativos de las asimetrías promedios de los grupos eran igualmente probables, y por lo tanto, debieran ocurrir en una razón de 1:1. La magnitud del tamaño del efecto y por ende de la asimetría del rasgo, se calculó de dos modos: (i) como el porcentaje del tamaño medio del rasgo (una medida escala independiente) dividiendo el valor medio del lado derecho menos el lado izquierdo (= Dif 1 ) por su valor promedio (Klingenberg et al. 1998), y (ii) como la diferencia Dif 1 estandarizada por la desviación estándar (DifStd).

\section{Efectos de la posición en la matriz}

Además de los análisis clásicos (ver arriba), se procedió complementariamente a aplicar el siguiente modelo de ANDEVA mixto de cuatro vías, completo o reducido si las interacciones de segundo o tercer orden no fueron significativas, para determinar si la asimetría bilateral puede ser afectada por el tipo de estrato. El modelo aplicado fue el siguiente:

$$
\begin{aligned}
Y_{m k l j k}= & \mu+L_{i}+E_{j}+S_{k}+I(E S)_{l(j k)}+L_{i j}+ \\
& L E_{i j}+L S_{i k}+E S_{j k}+L E S_{i j k}+ \\
& L I(E S)_{i l(j k)}+e_{m(l k i j)}
\end{aligned}
$$


donde, $Y_{m k l j k}$ es la $m$ medida repetida del índice $Y$ (variable respuesta) dentro del lado $i$ del individuo $l$ en el estrato $j$ en el sitio $k, \mu$ es la media poblacional, $L_{i}$ es el efecto fijo del lado $i, E_{j}$ es el efecto fijo del estrato j, $S_{k}$ es el efecto aleatorio del sitio k, I $(E S)_{l(j k)}$ es el efecto aleatorio del individuo $l$ dentro del estrato $\mathrm{j}$ y el sitio $\mathrm{k}$, $L I(E S)_{i l(j k)}$ es el efecto aleatorio del lado i con el individuo 1 dentro del estrato $\mathrm{j}$ y el sitio $\mathrm{k}$, $e_{m(l k i j)}$ es el error residual (varianza de las medidas repetidas) y los otros términos corresponden a las respectivas interacciones de segundo y tercer orden. Este ANDEVA mixto permite además poner a prueba si existen diferencias entre valores de los índices de forma asociados a estrato y a sitio o localidad, y si la asimetría direccional cambia con el estrato (interacción $L E)$. Previo a los análisis estadísticos se comprobó que se cumplieran las presunciones de normalidad y homogeneidad de varianzas de los residuos. Adicionalmente, se determinó si existía homogeneidad de varianzas del valor absoluto de (Dif 1) la diferencia entre medidas repetidas de cada valva e individuo (medida 1 medida 2) mediante una Prueba de Levene, para determinar el grado en que el EM podía inflar algunos de los estimadores del ANDEVA de cuatro vías usados (Palmer \& Strobeck 2003).

Puesto que el tamaño de los individuos en estratos inferiores de la matriz es más pequeño que en el estrato superior (Guiñez \& Castilla 1999), y aun cuando se seleccionó individuos dentro de rango de tallas comparables entre estratos (ver arriba), se puso a prueba si el promedio de la longitud máxima de los choritos difería entre estratos para las tres localidades. Por otra parte, en datos independientes obtenidos por Guiñez \& Castilla (1999) en Punta de Tralca, reanalizamos dos muestras de matrices para las que se disponía de información de la posición de los individuos y al mismo tiempo se disponía de datos de la longitud y altura máxima de las valvas que permitieron estimar al menos el índice de elongación (IELO), para reconfirmar si existía un patrón de cambio de la forma de la valva en matrices naturales en ambos estratos. Para ambos análisis se usó un ANDEVA de dos vías (estrato-factor fijo y localidad o muestra-factor aleatorio) con interacción. Finalmente, para determinar si los cambios en asimetría bilateral eran tamaño-dependientes se usó la prueba no paramétrica del coeficiente de correlación de rangos de Spearman entre el valor absoluto de Dif 1 para ambos índices versus el promedio de la longitud máxima para cada una de nuestras muestras (Palmer \& Strobeck 2003).

Todos los procedimientos y análisis estadísticos aplicados en este trabajo se corrieron usando SAS (SAS 1997). Para el caso de los ANDEVAS de dos vías y cuatro vías se usaron procedimientos de mínimos cuadrados (PROC GLM y opciones RANDOM/TEST y SLICE), y para el de cuatro vías se corrió además el modelo usando procedimientos de máxima verosimilitud restringida $($ REML $=$ Restricted Maximum Likelihood, PROC MIXED) para obtener los estimadores insesgados de varianza de los componentes aleatorios del modelo.

\section{RESULTADOS}

\section{Tipos de asimetría y error de medición}

Del total de 237 choritos seleccionados al azar que se trabajaron aquí, ocho individuos fueron considerados como extremos ("outliers") y no se incluyeron en los análisis que siguen. Las distribuciones de frecuencias de Dif 1 para todas las muestras fueron normales $(\mathrm{P}>0,15$; prueba de normalidad de Anderson-Darling) y sus varianzas homogéneas ( $\mathrm{P}>0,13$; prueba de Levene), por lo tanto los valores de curtosis no fueron significativamente distintos de cero, evidencia de que para ambos índices la distribución de Dif 1 fue unimodal y leptocúrtica. Lo anterior indica que no hay evidencia de antisimetría (platicurtosis: curtosis significativamente menor que cero). Por otra parte, las diferencias promedios entre la valva izquierda y la derecha para ambos índices de forma (IELO: elongación e IRED: redondez) y cada una de las localidades y estratos muestran en todos los casos la misma dirección: que las valvas derechas presentan mayores valores que las izquierdas (Dif $1>0$; Tabla 1A y 1B). También se observa que IELO es siempre menor en el estrato inferior que en el superior (Dif $2<0$, Tabla 1A) y para IRED esto es inverso (Dif $2>$ 0 , Tabla 1B). Estas tendencias son altamente significativas para cada rasgo por separado $(\mathrm{P}=$ 0,0156; prueba binomial) y para ambos rasgos en conjunto ( $\mathrm{P}<0,0002$; prueba binomial). La magnitud de las asimetrías en términos de rangos absolutos (Dif 1) variaron entre 0,004 y 
$0,045 \mathrm{~mm}$, y en términos del tamaño de los efectos muestra rangos como porcentaje del tamaño medio entre 2,55 y $0,57 \%$, y entre 0,255 y 0,070 como diferencia estandarizada (Tabla 1A y 1B). Esto sugiere que la asimetría de la forma es direccional (para ambos índices) con la valva derecha presentando mayores valores que la valva izquierda. Finalmente, respecto de la posible tamaño-dependencia de la asimetría direccional para IELO, se encontró que solo la muestra de Punta de Tralca mostraba una correlación negativa (sin realizar la corrección de Bonferroni) entre el índice y la longitud promedio $(\rho=-$ 0,59; $\mathrm{P}<0,001$; prueba de rangos de Spearman), las otras comparaciones fueron no significativas $(\mathrm{P}>0,42)$, y para todas las muestras combinadas la correlación fue no significativa $(\rho=-0,09 ; \mathrm{P}$ $>0,17)$. Para el índice IRED, la muestra de Punta de Tralca estrato superior mostró una correlación significativa $(\rho=-0,31 ; P=0,048)$, las otras muestras no fueron significativas $(\mathrm{P}>$ $0,31)$, y los datos combinados tampoco mostraron una correlación significativa $(\rho=-0,06 ; \mathrm{P}>$ 0,34). El ANDEVA aplicado para determinar si en nuestros datos existía evidencias de diferencia de tamaño entre estratos, muestra que la longitud máxima, como se esperaba, no difiere entre los estratos $\left(\mathrm{F}_{1,2}=13,63 ; \mathrm{P}=0,066\right)$.

TABLA 1

Promedio de los índices de elongación (A) y de redondez (B) para las valvas izquierda y derecha $( \pm \mathrm{EE})$. Se incluye el promedio de la diferencia entre valvas (Dif 1: valva derecha - valva izquierda) para ambos índices $( \pm \mathrm{EE})$, el tamaño del efecto expresado como porcentaje de Dif 1 con relación al promedio (\% Dif) y como la diferencia estandarizada en relación a la desviación estándar (DifStd), y la diferencia poblacional para valvas de cada lado entre estratos (Dif 2: estrato inferior - estrato superior)

Mean values for the elongation (A) and roundness (B) indexes for the left and right valves $( \pm$ SE). The mean differences between valves (Dif 1: Right valve minus Left valve) for both indexes $( \pm \mathrm{SE})$, the effect size estimated as the percentage of Dif 1 in relation to the mean, and the standardized difference (DifStd) in relation to the standard deviation, and the population difference for both valve sides between layer position (Dif 2: lower layer minus upper layer) are included

(A) Índice de elongación

\begin{tabular}{|c|c|c|c|c|c|c|c|}
\hline \multirow[t]{2}{*}{ Localidad } & \multirow[t]{2}{*}{$\mathrm{n}$} & & \multirow[t]{2}{*}{ Izquierda } & \multirow[t]{2}{*}{ Derecha } & \multirow[t]{2}{*}{ Dif 1} & \multicolumn{2}{|c|}{ Tamaño del efecto } \\
\hline & & & & & & $\%$ Dif & DifStd \\
\hline Punta de Tralca & & Dif 2: & $-0,109$ & $-0,078$ & & & \\
\hline Inferior & 31 & & $1,738 \pm 0,031$ & $1,783 \pm 0,032$ & $0,045 \pm 0,018$ & 2,55 & 0,255 \\
\hline Superior & 40 & & $1,847 \pm 0,021$ & $1,861 \pm 0,023$ & $0,014 \pm 0,014$ & 0,73 & 0,099 \\
\hline El Way & & Dif 2: & $-0,037$ & $-0,017$ & & & \\
\hline Inferior & 39 & & $1,769 \pm 0,028$ & $1,803 \pm 0,034$ & $0,034 \pm 0,014$ & 1,91 & 0,175 \\
\hline Superior & 40 & & $1,805 \pm 0,026$ & $1,820 \pm 0,027$ & $0,014 \pm 0,009$ & 0,80 & 0,088 \\
\hline Llacolén & & Dif 2: & $-0,113$ & $-0,107$ & & & \\
\hline Inferior & 40 & & $1,779 \pm 0,031$ & $1,800 \pm 0,031$ & $0,021 \pm 0,012$ & 1,20 & 0,111 \\
\hline Superior & 39 & & $1,892 \pm 0,033$ & $1,907 \pm 0,036$ & $0,015 \pm 0,012$ & 0,79 & 0,070 \\
\hline
\end{tabular}

(B) Índice de redondez

\begin{tabular}{|c|c|c|c|c|c|c|c|}
\hline \multirow[t]{2}{*}{ Localidad } & \multirow[t]{2}{*}{$\mathrm{n}$} & & \multirow[t]{2}{*}{ Izquierda } & \multirow[t]{2}{*}{ Derecha } & \multirow[t]{2}{*}{ Dif 1} & \multicolumn{2}{|c|}{ Tamaño del efecto } \\
\hline & & & & & & $\%$ Dif & DifStd \\
\hline Punta de Tralca & & Dif 2: & 0,017 & 0,012 & & & \\
\hline Inferior & 31 & & $0,748 \pm 0,012$ & $0,755 \pm 0,012$ & $0,007 \pm 0,007$ & 0,89 & 0,087 \\
\hline Superior & 40 & & $0,731 \pm 0,007$ & $0,742 \pm 0,006$ & $0,011 \pm 0,005$ & 1,50 & 0,238 \\
\hline El Way & & Dif 2: & 0,009 & 0,009 & & & \\
\hline Inferior & 39 & & $0,763 \pm 0,009$ & $0,768 \pm 0,008$ & $0,005 \pm 0,005$ & 0,62 & 0,078 \\
\hline Superior & 40 & & $0,755 \pm 0,009$ & $0,759 \pm 0,010$ & $0,004 \pm 0,006$ & 0,57 & 0,071 \\
\hline Llacolén & & Dif 2: & 0,022 & 0,021 & & & \\
\hline Inferior & 40 & & $0,772 \pm 0,009$ & $0,778 \pm 0,010$ & $0,006 \pm 0,006$ & 0,73 & 0,095 \\
\hline Superior & 39 & & $0,751 \pm 0,009$ & $0,757 \pm 0,009$ & $0,006 \pm 0,006$ & 0,78 & 0,106 \\
\hline
\end{tabular}


El ANDEVA mixto de dos vías aplicado indica que el error de medición no contribuye significativamente a ninguna forma de asimetría para IELO, puesto que el término de interacción de lado $\mathrm{x}$ individuo es significativamente distinto de cero ( $\mathrm{P}<0,001$; Tabla 2$)$. Sin embargo, para IRED solo para las muestras de Llacolén no habría una contribución del error de medición $(\mathrm{P}<0,002$; Tabla 2). Esto sugiere que el índice de redondez, no sería confiable para estudios de asimetría bilateral, por el mayor efecto del error de medición involucrado. Sin embargo, ambos índices serían confiables para la comparación de diferencias de forma entre individuos, pues para ambos el efecto de individuo es significativamente mayor que el error de medición $(\mathrm{P}<0,001$; Tabla 2$)$. En relación con los efectos del factor lado, para IELO e IRED y después de las correcciones de Bonferroni, el clásico ANDEVA muestra a muestra, no es capaz de detectar ningún efecto significativo para ninguna de las muestras $(\mathrm{P}>0,019$; $\mathrm{P}_{\text {crítica al } 5 \%}=0,0083$; Tabla 2).

\section{Efectos de la posición en la matriz}

En promedio, el índice de elongación es menor para el estrato inferior que para el superior (Dif $2<0$; Tabla 1A) e IRED muestra la tendencia contraria (Dif $2>0$; Tabla 1B; Fig. 1). Estas tendencias son estadísticamente significativas ( $\mathrm{P}$ $=0,0156$; prueba binomial), sugiriendo un efecto significativo del tipo de estrato. Por otra parte, se observa que para IELO la asimetría en el estrato inferior es siempre mayor que en el superior, el tamaño del efecto en términos de la diferencia estandarizada (DifStd; Tabla 1A) es entre $60 \%$ (Llacolén) y $157 \%$ (Punta de Tralca) mayor en el estrato inferior que en el superior (Fig. 1). Esto sugiere una posible interacción entre los efectos de posición (estrato) y asimetría (lado), incrementando la asimetría en el estrato inferior. En cambio, IRED no muestra una tendencia consistente en este aspecto (Tabla 1B).

La varianza de la asimetría de IELO, estimada como el valor absoluto de la diferencia entre medidas repetidas de cada valva e individuo ( $\mid$ medida 1 - medida $2 \mid$ ), difiere solo entre localidades $\left(\mathrm{F}_{2,455}=6,56 ; \mathrm{P}=0,002\right.$; prueba de Levene), y las varianzas para los efectos de lado, estrato e interacción de estrato x lado mostraron homogeneidad de varianzas $(\mathrm{P}>$ 0,132; prueba de Levene). Esto indicaría que solo el efecto de localidad podría estar enmascarado por el error de medición. En cambio para IRED se observa heterogeneidad de varianza para los efectos de localidad $\left(\mathrm{F}_{2,455}=\right.$ 12,30; $\mathrm{P}<0,001$; prueba de Levene), de estrato

TABLA 2

Análisis de varianza mixto de dos vías de los efectos de lado (fijo) e individuo (aleatorio) sobre los índices de elongación y redondez, para cada localidad y estrato. Los valores de significación estadística son los previos a las correcciones de Bonferroni

Two-way mixed analysis of variance of the effects of side (fixed) and individual mussel (random) on the elongation and roundness indexes for each locality and layer position. Significance values are before Bonferroni corrections

\begin{tabular}{|c|c|c|c|c|c|c|c|}
\hline \multirow{2}{*}{$\begin{array}{l}\text { Índice } \\
\text { Localidad }\end{array}$} & \multirow[t]{2}{*}{ Estrato } & \multicolumn{2}{|c|}{ Lado } & \multicolumn{2}{|c|}{ Individuo } & \multicolumn{2}{|c|}{ Lado $\mathrm{x}$ individuo } \\
\hline & & $\mathrm{F}$ & $\mathrm{P}$ & $\mathrm{F}$ & $\mathrm{P}$ & $\mathrm{F}$ & $\mathrm{P}$ \\
\hline \multicolumn{8}{|l|}{ Elongación } \\
\hline \multirow[t]{2}{*}{ Punta de Tralca } & Inferior & 5,979 & 0,021 & 10,937 & $<0,001$ & 34,214 & $<0,001$ \\
\hline & Superior & 1,006 & 0,322 & 9,310 & $<0,001$ & 16,347 & $<0,001$ \\
\hline \multirow{2}{*}{ El Way } & Inferior & 6,008 & 0,019 & 19,139 & $<0,001$ & 11,618 & $<0,001$ \\
\hline & Superior & 2,523 & 0,120 & 32,096 & $<0,001$ & 12,025 & $<0,001$ \\
\hline \multirow[t]{2}{*}{ Llacolén } & Inferior & 3,212 & 0,081 & 25,462 & $<0,001$ & 26,664 & $<0,001$ \\
\hline & Superior & 1,660 & 0,205 & 34,490 & $<0,001$ & 17,067 & $<0,001$ \\
\hline \multicolumn{8}{|l|}{ Redondez } \\
\hline \multirow{2}{*}{ Punta de Tralca } & Inferior & 0,936 & 0,341 & 10,968 & $<0,001$ & 0,463 & 0,988 \\
\hline & Superior & 4,529 & 0,040 & 5,791 & $<0,001$ & 1,690 & 0,024 \\
\hline \multirow[t]{2}{*}{ El Way } & Inferior & 0,863 & 0,359 & 9,847 & $<0,001$ & 0,529 & 0,984 \\
\hline & Superior & 0,469 & 0,498 & 7,381 & $<0,001$ & 1,906 & 0,008 \\
\hline \multirow[t]{2}{*}{ Llacolén } & Inferior & 0,935 & 0,340 & 8,859 & $<0,001$ & 2,983 & $<0,001$ \\
\hline & Superior & 0,965 & 0,332 & 7,079 & $<0,001$ & 2,190 & 0,002 \\
\hline
\end{tabular}


$\left(\mathrm{F}_{1,456}=40,77 ; \mathrm{P}<0,001\right)$ y de la interacción lado $\mathrm{x}$ estrato $\left(\mathrm{F}_{3,454}=13,60 ; \mathrm{P}<0,001\right)$. En conclusión, para el análisis que sigue IELO es confiable de no presentar diferencias que puedan surgir como un artefacto del error de medición, en particular debido a que el efecto de localidad se considera aleatorio; esto es diferente para IRED para el que se deben tener extremas precauciones en la interpretación de sus resultados.

El ANDEVA mixto de cuatro vías (lado, estrato, localidad e individuo) completo, aplicado a ambos índices no mostró efectos significativos para las interacciones de segundo y tercer orden que incluían el efecto de localidad (o sitio), por lo que los resultados que se muestran para el modelo reducido no incluyen esas interacciones, se conservó sí, el efecto principal de localidad considerándolo como bloque aleatorio. Para el índice IELO fueron significativos los efectos de lado ( $\mathrm{P}<0,001$; Tabla 3A) Estra-
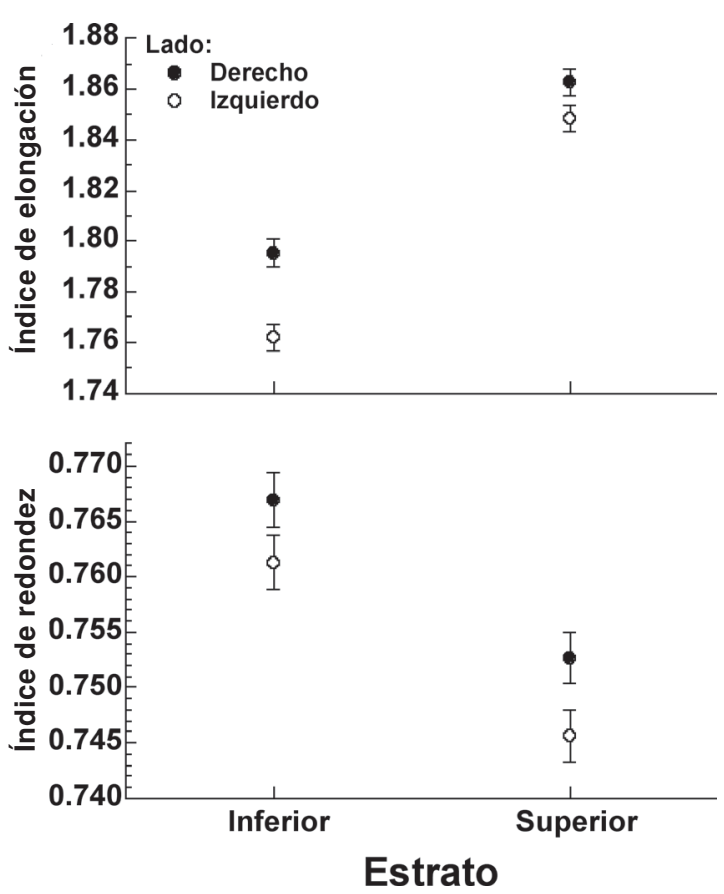

Fig. 1: Promedio ( \pm EE) de los índices de forma de elongación (IELO) y de redondez (IRED), para las valvas derecha e izquierda en relación con el estrato (inferior o superior) en que se encuentran los choritos en las matrices.

Mean values $( \pm \mathrm{SE}$ ) of the elongation (IELO) and roundness (IRED) shape indexes, for the right and left shell valve in relation to the spatial position of the mussels in the layer matrices (lower or upper stratum). to $(\mathrm{P}=0,002$; Tabla $3 \mathrm{~A})$, individuo dentro de localidad $(\mathrm{P}<0,001$; Tabla $3 \mathrm{~A})$ y la interacción de lado $\mathrm{x}$ individuo dentro de localidad $(\mathrm{P}<$ 0,001; Tabla 3A). Sin embargo, no se observaron diferencias significativas del efecto de localidad ( $\mathrm{P}=0,243$; Tabla $4 \mathrm{~A})$, ni para la interacción de lado por estrato $(\mathrm{P}=0,086$; Tabla 4A). Esto confirma estadísticamente la existencia de $\mathrm{AD}$ y el cambio de elongación entre estratos, pero no la interacción de estrato $\mathrm{x}$ lado (asimetría). Sin embargo, al aplicar un análisis de tajada ("SLICE") al término de interacción (PROC GLM/LSMEANS OPTION; SAS 1997) encontramos que para el estrato inferior la diferencia entre lados (= asimetría) fue estadísticamente significativa $\left(\mathrm{F}_{1,227}=18,22 ; \mathrm{P}<0,001\right)$, pero no fue significativa para el estrato superior $\left(\mathrm{F}_{1,227}=3,83 ; \mathrm{P}=0,052\right)$. Los datos de muestras independientes de choritos, reanalizados de Guiñez \& Castilla (1999), confirman estos resultados en el sentido que los individuos de capas inferiores presentan un índice de elongación significativamente más pequeño (promedio $=1,543 ; \mathrm{SE}=0,027 ; \mathrm{F}_{1,92}=29,02$; $\mathrm{P}<0,001)$ que aquellos del estrato superior (promedio $=1,759, \mathrm{SE}=0,025)$. En conjunto estos resultados indican que los choritos tienden a ser significativamente menos elongados en estratos inferiores, pero que al mismo tiempo incrementan la asimetría direccional, en relación con los choritos del estrato superior de la matriz. Por otra parte, una estimación de los componentes de varianza (REML) de los factores aleatorios del modelo, sobre IELO, mostró varianzas significativamente distintas de cero $(\mathrm{P}<0,001)$ para los componentes de: error de medición (1,1\% error residual), individuo dentro de localidad y estrato $(89,5 \%)$ e interacción de lado $\mathrm{x}$ individuo dentro de localidad y estrato $(8,9 \%)$, en cambio la varianza de localidad no fue distinta de cero $(\mathrm{P}=0,756)$, por lo que el error de medición efectivamente es mucho menor que la varianza del efecto de individuo y de lado $\mathrm{x}$ individuo.

Para IRED el ANDEVA de cuatro vías mostró diferencias significativas tanto para los efectos de lado ( $\mathrm{P}=0,008$; Tabla 3 ) como para los efectos de estrato $(\mathrm{P}=0,002$; Tabla 3$)$, localidad ( $\mathrm{P}=0,041$; Tabla 3$)$ e individuo dentro de localidad y estrato $(\mathrm{P}<0,001)$, no siendo significativos los efectos de interacción de lado $\mathrm{x}$ estrato $\mathrm{y}$ lado $\mathrm{x}$ individuo dentro de localidad y estrato $(\mathrm{P}>0,248$; Tabla 3$)$. Esto sugiere 
también AD asociado a IRED. Sin embargo, los componentes de estrato, localidad y la interacción lado x estrato podrían estar inflados por el error de medición. La estimación de los componentes de varianza (REML) de IRED mostró que son significativamente diferentes de cero $(\mathrm{P}<0,001)$ las varianzas de los componentes del error residual $(33,2 \%)$ y de individuo dentro de localidad y estrato $(65,4 \%)$. En cambio, el efecto de localidad $(2,2 \%)$ y de la interacción de lado $\mathrm{x}$ individuo dentro de localidad y estrato $(1,3 \%)$ no son significativamente distintas de cero $(\mathrm{P}>0,49)$. Esto confirma que para IRED el error de medición es extremadamente alto $(33,2 \%)$.

\section{DISCUSIÓN}

Nosotros hemos aportado evidencias de que ambos índices de forma (IELO e IRED) cambian con la posición dentro de la matriz y al mismo tiempo muestran una asimetría direccional. Sin embargo, IRED no es un rasgo que pueda ser de utilidad para estudios de asimetría fluctuante o direccional, puesto que se encuentra fuertemente influenciado por el error de medición. Por el contrario, IELO presenta una baja influencia del error de medición y, por consecuencia, es un índice confiable para estudios posteriores de la forma y su relación con la asimetría bilateral. Ambos índices mostraron tendencias similares pero de signo opuesto, por ejemplo, en sus tendencias asociadas con los valores de la diferencia promedio entre estratos (Dif 2, Tabla 1), lo que sugiere que debieran estar correlacionados negativamente. En efecto, la correlación de rangos de Spearman aplicada sobre los promedios de ambos rasgos para cada individuo fue significativa y negativa $(\rho=$ - 0,78; P < 0,001). Es esperable que cuando los individuos son menos elongados al mismo tiempo tiendan a ser más redondeados, por lo mismo se debiera esperar que su tendencia a ser más asimétricos estuviera también correlacionada. La correlación de Spearman de la diferencia individual entre ambas valvas (Ind 1) no fue significativa $(\rho=-0,02 ; \mathrm{P}=0,71)$, lo que po-

TABLA 3

Análisis de varianza mixto de cuatro vías de los efectos de lado (fijo), estrato (fijo), localidad (aleatorio) e individuo (aleatorio) sobre los índices de elongación (A) y redondez (B): gl num y gl den $=$ grados de libertad del numerador y denominador, respectivamente; $\mathrm{F}=$ razón de $\mathrm{F} ; \mathrm{P}>\mathrm{F}=$ probabilidad no ajustada; SC Tipo III y SC Den = sumatorias de cuadrados tipo III del numerador y denominador, respectivamente

Four-way mixed analysis of variance of the effects of side (fixed), layer position (fixed), locality (randon) and mussel individual (random) on the elongation (A) and roundness (B) indexes: gl num and gl den = degrees of freedom of the numerator and denominator, respectively; $\mathrm{F}=\mathrm{F}$ ratio; $\mathrm{P}>\mathrm{F}=$ non-adjusted probability; SC tipo III and SC den $=$ type III mean squares of the numerator and denominator, respectively

(A) Índice de elongación

\begin{tabular}{|c|c|c|c|c|c|c|}
\hline Fuente de variación & GL Num & GL Den & SC Tipo III & SC Den & $\mathrm{F}$ & $\mathrm{P}>\mathrm{F}$ \\
\hline Lado & 1 & 227 & 0,1258 & 0,0064 & 19,66 & $<0,001$ \\
\hline Estrato & 1 & 225 & 13,226 & 0,1276 & 10,66 & 0,002 \\
\hline Lado $\mathrm{x}$ estrato & 1 & 227 & 0,0189 & 0,0064 & 2,96 & 0,086 \\
\hline Loc & 2 & 225 & 0,1817 & 0,1276 & 1,42 & 0,243 \\
\hline Ind (Loc $\mathrm{x}$ estrato) & 225 & 227 & 0,1276 & 0,0064 & 19,936 & $<0,001$ \\
\hline Lado $x$ Ind (Loc $x$ estrato) & 227 & 458 & 0,0064 & 0,0004 & 17,308 & $<0,001$ \\
\hline
\end{tabular}

(B) Índice de redondez

\begin{tabular}{lrrrrrr}
\hline Fuente de variación & GL Num & GL Den & SC Tipo III & SC Den & F & P > F \\
\hline Lado & 1 & 227 & 0,0092 & 0,0013 & 7,112 & 0,008 \\
Estrato & 1 & 225 & 0,0513 & 0,0108 & 4,755 & 0,030 \\
Lado x estrato & 1 & 227 & 0,0001 & 0,0013 & 0,097 & 0,752 \\
Loc & 2 & 225 & 0,0349 & 0,0108 & 3,237 & 0,041 \\
Ind (Loc x estrato) & 225 & 227 & 0,0108 & 0,0013 & 8,294 & $<0,001$ \\
Lado x Ind (Loc x estrato) & 227 & 458 & 0,0013 & 0,0012 & 1,079 & 0,248 \\
\hline
\end{tabular}


dría ser explicado por la influencia del error experimental en la estimación de IRED, o porque los mecanismos subyacentes y causales de la $\mathrm{AD}$ no son los mismos para ambos rasgos.

En el chorito $M$. edulis se ha demostrado que existe una fuerte influencia entre la forma de la valva y la densidad poblacional, observándose un incremento de la forma elongada al aumentar la densidad (L/H) (Seed 1968, Alunno-Bruscia et al. 2001). En el chorito Geukensia demissa se han comunicado distorsiones severas en la forma de las valvas en densidades extremadamente altas (Bertness \& Grosholz 1985). Aunque en $M$. edulis existen evidencias de que la posición de los individuos en agregaciones, en términos de borde versus centro, influencia su crecimiento y potencial reproductivo (Okamura 1986), y que en $P$. purpuratus los individuos ubicados en estratos inferiores presentan menores tamaños (Alvarado \& Castilla 1996, Guiñez \& Castilla 1999), en relación con los del estrato superior, en este trabajo demostramos que los choritos en distintos estratos de la matriz cambian de forma. La densidad poblacional puede alterar la forma de los choritos a través de la competencia por espacio (interferencia) debido a efectos mecánicos asociados a la compresión física de los organismos circundantes o por competencia por alimento (explotación) (Seed 1968, Alunno-Bruscia et al. 2001). Sin embargo, no existe aún una explicación causal de cómo la mayor o menor disponibilidad de alimento puede afectar la forma, la evidencia experimental para este efecto ha sido recientemente documentada (Alunno-Bruscia et al. 2001). Ambos componentes de la competencia pueden explicar el cambio de forma aquí documentado. Sin embargo, la evidencia experimental y de campo en $P$. purpuratus (R. Guiñez, resultados no publicados) sugieren que la densidad per se tiene efectos distintos a la estratificación (número de estratos) (Guiñez \& Castilla 1999, 2001) sobre la forma. En efecto, cuando se controló la densidad y se varió la estratificación, los choritos se hicieron menos alongados, no encontrándose un efecto significativo de la densidad cuando se controló la estratificación (R. Guiñez, resultados no publicados). La explicación causal de estos hallazgos está abierta a la investigación. Sin embargo, los resultados experimentales dan respaldo a proposiciones teóricas y experimentales de campo, que sugieren que la competencia intraespecífica en $P$. purpuratus y el autorraleo (self-thining) están determinados por la estratificación, más que por la densidad (Guiñez \& Castilla 1999).

Las comparaciones para ambos índices de forma, a juzgar por los valores de tamaño de los efectos, por las pruebas binomiales y el ANDEVA mixto de cuatro vías usado, muestran diferencias significativas entre ambas valvas (Dif 1, Tabla 1) y por tanto, la presencia significativa de $\mathrm{AD}$ aunque de efecto pequeño. Pero los análisis estándar o clásicos de ANDEVA de dos vías muestra a muestra, sugeridos en la literatura (Palmer \& Strobeck 1986, Palmer 1994, Palmer \& Strobeck 2003), no permitieron demostrar diferencias significativas asignables a AD. Esto significa que de acuerdo a este enfoque clásico, nosotros debimos haber concluido erróneamente que nuestros datos confirmaban la presencia solo de asimetría fluctuante y no de asimetría direccional. Por lo que sugerimos la implementación, en este tipo de estudios, del uso de análisis de varianza más complejos que aquellos comúnmente usados en la literatura. Sin embargo, nuestros datos son consistentes para concluir que ambos rasgos presentan $\mathrm{AD}$ de efecto pequeño, probablemente respondiendo de manera distinta al estrés representado por la posición en la matriz. Por otra parte, los choritos de los estratos inferiores son significativamente menos elongados y muestran una mayor $\mathrm{AD}$ en los estratos inferiores que en el estrato superior.

Dos estudios recientes en bivalvos encuentran evidencia de asimetría fluctuante de rasgos de las valvas (Fréchette \& Daigle 2002, Fréchette et al. 2003). En el primero, se encuentra que la sobrevivencia decrece con la AF de las orejas de la valva en el pectínido Chlamys islandica (Fréchette \& Daigle 2002), y en el segundo, se encuentra diferencias significativas en la FA del peso de las valvas en ostras Crassostrea gigas, entre individuos muertos y vivos (Fréchette et al. 2003). Sin embargo, en este trabajo y por primera vez en la literatura de moluscos (al menos conocido por los autores) se sugiere la existencia de AD modulada por la estratificación (posición en la matriz).

Estos primeros resultados de nuestra investigación pueden permitir avanzar tanto en la investigación experimental de los mecanismos subyacentes de la asimetría direccional y fluctuante en bivalvos, como en los mecanismos ecológicos y fisiológicos que los determinan, 
en particular relacionados con la vida gregaria, su expresión en el ámbito geográfico de distribución de la especie y su posible implicancia evolutiva.

\section{AGRADECIMIENTOS}

Se agradece a G. Peralta y L. Prado por el apoyo en el trabajo en terreno. Paula Neill tradujo el resumen al inglés. A los revisores anónimos que permitieron mejorar la redacción final y enfoque del manuscrito. Este trabajo fue financiado por el proyecto FONDECYT 1020484 a RG, y parcialmente por el Centro de Estudios Avanzados en Ecología \& Biodiversidad (Programa 6) Proyecto 1501-0001. R. Guiñez agradece el apoyo de Sergio Navarrete y Juan Carlos Castilla a través del Proyecto Mellon FoundationPontificia Universidad Católica de Chile.

\section{LITERATURA CITADA}

ALUNNO-BRUSCIA M, E BOURGET \& M FRÉCHETTE (2001) Shell allometry and length-mass-density relationship for Mytilus edulis in an experimental food-regulated situation. Marine Ecology Progress Series 219: 177-188

ALVARADO JL \& JC CASTILLA (1996) Tridimensional matrices of Perumytilus purpuratus on intertidal platforms with varying wave forces in central Chile. Marine Ecology Progress Series 133: 135-141.

BEARDMORE JA (1960) Development instability in constant and fluctuating temperatures. Heredity 14 : 411-422.

BERTNESS MD \& ED GROSHOLZ (1985) Population dynamics of the ribbed mussel, Geukensia demissa: The costs and benefits of an aggregated distribution. Oecologia 67: 192-204.

BERTNESS MD, SD GAINES \& SM YEH (1998) Making mountains out of barnacles: The dynamics of acorn barnacle hummocking. Ecology 79: 1382-1394.

CASTILLA JC (1981) Perspectivas de investigación en estructura y dinámica de comunidades intermareales rocosas de Chile central. II. Depredadores de alto nivel trófico. Medio Ambiente (Chile) 5: 190-215.

CASTILLA JC, R GUIÑEZ, JL ALVARADO, C PACHECO \& M VARAS (2000) Distribution, population structure, population biomass and morphological characteristics of the tunicate Pyura stolonifera in the bay of Antofagasta, Chile. Marine Ecology-Pubblicazioni della Stazione Zoologica di Napoli 21: 161-174.

CHINZEI K, E SAVAZZI \& A SEILACHER (1982) Adaptational strategies of bibalves living as infaunal secondary soft bottom dwellers. Neues Jahrbuch fuer Geologie und Palaeontologie, Abhandlungen 164: 229-244.

CIGARRÍA J \& J FERNÁNDEZ (1998) Manila clam (Ruditapes philippinarum) culture in oyster bags: influence of density on survival, growth and biometric relationships. Journal of the Marine
Biological Association, United Kingdom 78: 551560.

DITCHKOFF SS, RL LOCHMILLER, RE MASTERS, WR STARRY \& DM LESLIE JR (2001) Does fluctuating asymmetry of antlers in white-tailed deer (Odocoileus virginianus) follow patterns predicted for sexually selected traits? Proceedings of the Royal Society of London, Biological Sciences 268: 891-898.

FRÉCHETTE M, P GOULLETQUER \& G DAIGLE (2003) Fluctuating asymmetry and mortality in cultured oysters (Crassostrea gigas) in MarennesOleron basin. Aquatic Living Resources 16: 339346.

FRÉCHETTE M \& D LEFAIVRE (1990) Discriminating between food and space limitation in benthic suspension feeders using self-thinning relationships. Marine Ecology Progress Series 65: 15-23.

FRÉCHETTE M \& G DAIGLE (2002) Growth, survival and fluctuating asymmetry of Iceland scallops in a test of density-dependent growth in a natural bed. Journal of Experimental Marine Biology and Ecology 270: 73-91.

GEST TR, MI SIEGEL \& J ANISTRANSKI (1986) The long bones of neonatal rats stressed by cold, heat, and noise exhibit increased fluctuating asymmetry. Growth 50: 385-389.

GRAHAM JH, DC FREEMAN \& JM EMLEN (1993) Developmental stability: a sensitive indicator of populations under stress. En: Landis Js, Js Hughes \& Ma Lewis (eds) Environmental toxicology and risk assessment: 136-158. ASTM STP 1179, ASTM, Philadelphia, Pennsylvania, USA.

GUIÑEZ R (1996) Dinámica poblacional del chorito maico, Perumytilus purpuratus (Lamarck 1819) (Bivalvia: Mytilidae), en gradientes de exposición al oleaje. Ph. D. Thesis, Pontificia Universidad Católica de Chile, Santiago, Chile. 213 pp.

GUIÑEZ R \& JC CASTILLA (1999) A tridimensional self-thinning model for multilayered intertidal mussels. American Naturalist 154: 341-357.

GUIÑEZ R \& JC CASTILLA (2001) An allometric tridimensional model of self-thinning for a gregarious tunicate. Ecology 82: 2331-2341.

HOSKEN DJ, WU BLANCKENHORN \& PI WARD (2000) Developmental stability in yellow dong flies (Scathophaga stercoraria): fluctuating asymmetry, heterozygosity and environmental stress. Journal of Evolutionary Biology 13: 919-926.

KLINGENBERG CP, GS MCINTYRE \& SD ZAKLAN (1998) Left-right asymmetry of fly wings and the evolution of body axes. Proceedings of the Royal Society of London, Biological Sciences 265: 12551259.

KRAAK SBM (1997) Fluctuating around directional asymmetry? Trends in Ecology \& Evolution 12: 230.

LEARY RF \& FW ALLENDORF (1989) Fluctuating asymmetry as an indicator of stress in conservation biology. Trends in Ecology \& Evolution 4: 214217.

LENS L \& S VAN DONGEN (2000) Fluctuating and directional asymmetry in natural bird populations exposed to different levels of habitat disturbance, as revealed by mixture analysis. Ecology Letters 3: 516-522.

LEUNG B, MR FORBES \& D HOULE (2000) Fluctuating asymmetry as a bioindicator of stress: comparing efficacy of analyses involving multiple traits. American Naturalist 155: 101-115. 
MØLLER AP (1997) Development stability and fitness: a review. American Naturalist 149: 916-932.

OKAMURA B (1986) Group living and effect of spatial position in aggregations of Mytilus edulis. Oecologia 69: 341-347.

PAINE RT \& TH SUCHANEK (1983) Convergence of ecological processes between independently evolved competitive dominants; a tunicate-mussel comparison. Evolution 37: 821-831.

PALMER AR (1994) Fluctuating asymmetry analyses: a primer. En: Markow TA (ed) Developmental instability: its origins and evolutionary implications: 335-364. Kluwer, Dordrecht, The Netherlands.

PALMER AR (1996a) Waltzing with asymmetry. BioScience 46: 518-532.

PALMER AR (1996b) From symmetry to asymmetry: phylogenetic patterns of asymmetry variation in animals and their evolutionary significance. Proceedings of the National Academy of Sciences USA 93: $14279-14286$.

PALMER AR \& C STROBECK (1986) Fluctuating asymmetry: measurement, analysis, patterns. Annual Review of Ecology and Systematics 17: 391-421.

PALMER AR \& C STROBECK (1992) Fluctuating asymmetry as a measure of development stability: implications of non-normal distributions and power

Editor Asociado: Rodrigo Medel

Recibido el 3 de mayo de 2004; aceptado el 13 de noviembre de 2004 of statistical tests. Acta Zoologica Fennica 191: 57 72.

PALMER AR \& C STROBECK (2003) Fluctuating asymmetry analyses revisited. En: Polak M (ed) Development instability. Causes and consequences: 279-319. Oxford University Press, Oxford, United Kingdom.

PITHER J \& PD TAYLOR (2000) Directional and fluctuating asymmetry in the black-winged damselfly Calopteryx maculata (Beauvois) (Odonata: Calopterygidae). Canadian Journal of Zoology 78: 1740-1748.

QUINN GP \& MJ KEOUGH (2002) Experimental design and data analysis for biologists. Cambridge University Press, Cambridge, United Kingdom. 537 pp.

SAS (1997) SAS/STAT software: changes and enhancements through release 6.12. SAS Institute, Cary, North Carolina, USA. 1,162 pp.

SEED R (1968) Factors influencing shell shape in the mussel Mytilus edulis. Journal of the Marine Biological Association of the United Kingdom 48: 561-584.

SUCHANEK TH (1986) Mussels and their role in structuring rocky shore communities. En: Moore PG \& R Seed (eds) The ecology of rocky coasts: 70-96. Columbia University Press, New York, New York, USA. 